Nervenarzt 2018 $89: 747-753$

https://doi.org/10.1007/s00115-018-0529-x

Online publiziert:23. Mai 2018

(c) Der/die Autor(en) 2018

CrossMark

 \\ ' Deutsches Resilienz Zentrum (DRZ) Mainz, Mainz, Deutschland \\ ${ }^{2}$ Klinik für Psychiatrie und Psychotherapie, Universitätsmedizin Mainz, Mainz, Deutschland \\ ${ }^{3}$ Neuroimaging Center Mainz (NIC), Forschungszentrum Translationale Neurowissenschaften (FTN), \\ Universitätsmedizin Mainz, Mainz, Deutschland
}

\title{
Aktuelle Konzepte der Resilienzforschung
}

jedoch nicht gesichert [2]. Zudem konnte diese Sichtweise keinen substanziellen Beitrag zur Erklärung des Resilienzphänomens leisten [1].

In den letzten Jahren wird Resilienz vor allem als Ergebnis bzw. Produkt am Ende des Anpassungsprozesses an Stressoren verstanden $[12,14,15]$. Resilienz als Ergebnis wird vermutlich durch eine Vielzahl (neuro-)biologischer, psychologischer und sozialer Ressourcen (sog. Resilienzfaktoren) beeinflusst. In diesem Sinne könnten auch natürliche Prädispositionen bzw. Persönlichkeitseigenschaften als Teil einer Vielzahl von Resilienzfaktoren angesehen werden, welche die Anpassung an Stressoren positiv beeinflussen und Resilienz partiell determinieren $[14,15]$.

\section{Der Resilienzbegriff im Wandel}

Der aus dem Lateinischen („resilire“) abgeleitete Begriff Resilienz (deutsch: abprallen, zurückspringen) wurde in den vergangenen Jahrzehnten von verschiedenen Disziplinen aufgegriffen [1]. Psychische Resilienz bezeichnet die Aufrechterhaltung bzw. rasche Wiederherstellung der psychischen Gesundheit während oder nach stressvollen Lebensumständen [14, 15].

Die Definition und folglich auch die Operationalisierung und Erfassung von Resilienz sind sehr heterogen (z. B. [23]). Dies ist auch auf den stetigen Wandel des Konzepts in den letzten 30 Jahren zurückzuführen [12, 14, 15].Zu Beginn der Resilienzforschung wurde Resilienz überwiegend als natürliche Veranlagung bzw. stabile Persönlichkeitseigenschaft definiert [15]. Die empirische Evidenz hierfür ist

\section{Resilienz als Ansatz der Gesundheitsförderung}

Die heutige Resilienzforschung ist das Resultat eines Paradigmenwechsels: von der krankheitsorientierten Pathogenese und der Untersuchung von Ursachen und Behandlungsmöglichkeiten psychischer Erkrankungen hin zur ressourcenorientierten Salutogenese und der Ermittlung von Schutzmechanismen [1]. Als Konzept der Gesundheitsförderung [24] stellt die Resilienzforschung einen übergeordneten Ansatz dar. Sie grenzt sich von der traditionellen pathophysiologischen Forschung ab bzw. ergänzt diese, indem sie zeitlich vor der Entstehung psychischer Erkrankungen ansetzt [15]. Zugleich verwendet die Resilienzforschung im Allgemeinen eine breitere Vorgehensweise als bisherige Präventionsansätze. Anstelle der Vorbeugung umschriebener Erkrankungen (z. B. Depression) und der Krankheitsforschung liegt der Fokus vielmehr aufder diagnoseunspezifischen $\mathrm{Ge}$ sundheitsforschung und -förderung angesichts von Stressoren [15]. Im Folgenden werden aktuelle Vorschläge zur Operationalisierung von Resilienz in empirischen Studien vorgestellt.

\section{Resilienzfaktoren, Mecha- nismen und Resilienz}

Bislang wurden zahlreiche potenzielle Resilienzfaktoren untersucht, die in verschiedenen Populationen mit der erfolgreichen Anpassung an einen Stressor assoziiert sind $[1,11]$. Es wird angenommen, dass sowohl interne (z. B. Bewältigungsstile, kognitive Fähigkeiten, [Epi-] 


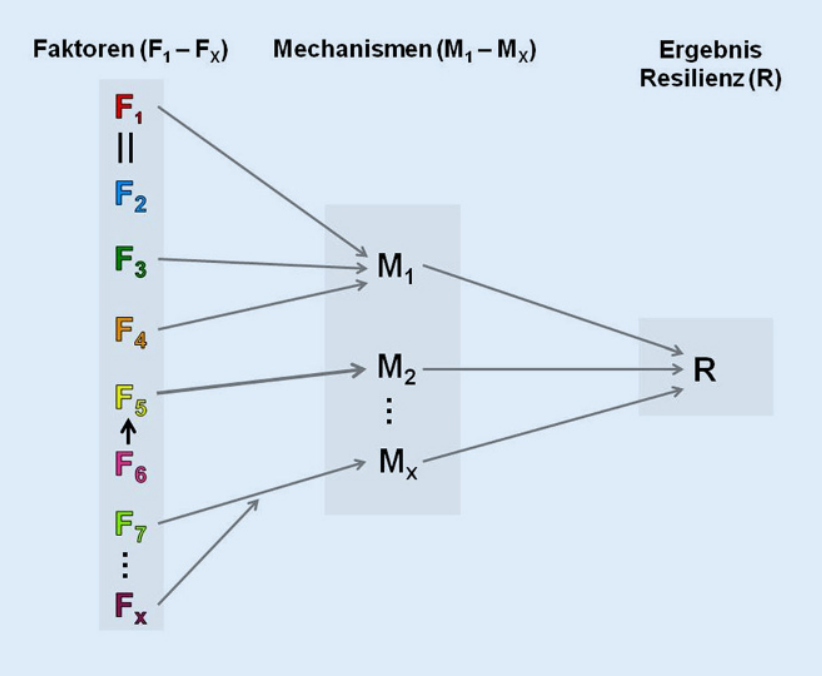

Abb. $1 \triangleleft$ Resilienzfaktoren $\left(\mathrm{F}_{1}-\mathrm{F}_{\mathrm{X}}\right)$, Resilienzmechanismen $\left(M_{1}-M_{x}\right)$ und Resilienz (R) (Adaptiert nach Kalisch et al. [14], mit freundl. Genehmigung Cambridge University Press)
Genetik) als auch externe Ressourcen (z.B. sozioökonomischer Status, soziale Unterstützung) Resilienz als Ergebnis begünstigen [14]. Prospektive Studien zur Untersuchung der komplexen Zusammenhänge dieser Faktoren mit Resilienz, in denen auch die Stressorexposition berücksichtigt wird, sind allerdings weniger vorhanden [15]. Zudem ist zu beachten, dass die Faktoren im Einzelnen häufig nur einen geringen Teil der Varianz in der Anpassung an Stressoren erklären und ihre Vorhersagekraft für Resilienz, z.B. über verschiedene Populationen hinweg, aktuell uneindeutig ist [15]. Gleichzeitig ist bekannt, dass viele der Resilienzfaktoren miteinander assoziiert sind oder interagieren [14]. Inzwischen wird daher diskutiert, dass die Wirkung der Faktoren auf Resilienz über eine wahrscheinlich geringere Anzahl übergeordneter, kognitiver und neuronaler Mechanismen (sog. Resilienzmechanismen) im Sinne gemeinsamer Wirkpfade vermittelt werden könnte $[14,16]$.

\section{I) Neurale/kognitive Resilienzmechanismen wirken als Mediatoren}

Die „positive appraisal style theory of resilience" (PASTOR) postuliert, dass ein positiver Bewertungsstil im Falle potenziell bedrohlicher oder negativer Reize und Situationen einen solchen Mechanismus darstellt und die positive Wirkung verschiedener Resilienzfaktoren auf Re- silienz mediiert [14]. So könnte z. B. eine hohe soziale Unterstützung (ein häufig genannter Resilienzfaktor) dazu beitragen, dass eine Person Stressoren positiver (um-)bewertet. In der Folge wird Resilienz gefördert, was sich auch in einer geringeren Anfälligkeit der Person für stressbedingte Erkrankungen äußert. Daneben sind weitere Mechanismen auf verschiedenen Ebenen (z. B. neurobiologisch, psychologisch) denkbar, die jedoch alle auf der Ebene des Individuums bzw. der Funktion seines Gehirns/Geistes angesiedelt sind. - Abb. 1 veranschaulicht die Differenzierung zwischen Resilienzfaktoren und -mechanismen sowie Resilienz als Ergebnis.

\section{Transdiagnostische, multi- systemische und quantitative Operationalisierung}

In der aktuellen Forschung wird Resilienz überwiegend dichotom operationalisiert. Untersucht wird die Frage, warum manche Menschen eine spezifische Erkrankung (z. B. Depression) nicht entwickeln, obwohl sie mit identischen oder ähnlichen Stressoren konfrontiert werden, wie Personen, die an einer derartigen psychischen Folgeerkrankung leiden [14]. Das Heranziehen eines kategorialen diagnostischen Klassifikationssystems (krank vs. gesund) bei der Operationalisierung von Resilienz birgt jedoch verschiedene Probleme. Dem Umstand, dass psychische Symptome zwischen verschiedenen Störungsbildern überlappen und psychische
Erkrankungen häufig komorbid auftreten, wird so nicht ausreichend Genüge getan [14]. „Gesunde“ und „kranke“ Personen sind meist nicht klar voneinander abgrenzbar und traditionelle Krankheitskategorien erschweren die Erforschung zugrunde liegender (z. B. auch neurobiologischer) (Schutz-)Mechanismen. Auch die neurobiologischen und behavioralen Dysfunktionen (z. B. Hypervigilanz), die psychischen Störungen zugrunde liegen, werden durch die Dichotomisierung unzureichend berücksichtigt [14].

Kalisch et al. [14] schlagen daher einen transdiagnostischen Ansatz vor. Demnach sollte sich die Erforschung von Resilienz und ihrer Mechanismen nicht auf Erkrankungen, sondern auf Dysfunktionen (z.B. Anhedonie, hyperimpulsives Verhalten) fokussieren. Anstelle der Suche nach erkrankungsspezifischen Resilienzmechanismen, die lediglich vor einer bestimmten psychischen Störung schützen (z.B. Depression), sollten vielmehr dysfunktionsspezifische Mechanismen identifiziert werden, die vor der stressinduzierten Beeinträchtigung in einer Funktionsdimension (z.B. Emotionsregulation, Impulskontrolle) schützen.

Im nächsten Schritt postulieren Kalisch et al. [13, 14] eine multisystemische Resilienzforschung. Da stressbedingte Dysfunktionen i.d.R. nicht einzeln auftreten, stellt sich die Frage nach der Existenz übergeordneter (genereller) Resilienzmechanismen, die vor mehr als einer Dysfunktion schützen. Beispielsweise könnte ein Resilienzmechanismus, der nach dem Ende eines Stressors die hierdurch ausgelöste Reaktion der Hypothalamus-Hypophysen-Nebennierenrinden(HHN)-Achse rechtzeitig beendet, vor mehreren stressbedingten Dysfunktionen schützen, die aus einer langandauernden Aktivität der HHNAchse und einer zu hohen Ausschüttung des Hormons Kortisol resultieren [13].

Da psychische Symptome oder Dysfunktionen nicht dichotom auftreten (z.B. jemand ist nicht entweder impulsiv oder nicht), erscheinen Schwellenwerte für die Annahme einer Dysfunktion als pathologisch eher ungeeignet [13]. Daher schlagen Kalisch et al. [13, 14] die Verwendung quantitativer (kontinu- 
Hier steht eine Anzeige.




ierlicher) Zielvariablen vor. Zur Messung einer psychischen Dysfunktion könnte ein Score, z. B. aus einem geeigneten Fragebogenmaß oder einem behavioralen oder (neuro)physiologischen Funktionsmaß, gebildet werden. Ist das Ziel, nicht nur dysfunktionsspezifische, sondern generelle Resilienzmechanismen zu identifizieren, böte es sich an, einen globalen Score der psychischen Dysfunktion zu bilden, indem quantitative Scores in mehreren Funktionsdimensionen (z.B. somatische Dysfunktion, depressive Symptome, Angst) summiert oder gemittelt werden. Auf diese Weise ließen sich quantitative Aussagen über die Veränderung psychischer Dysfunktionen zwischen zwei oder mehreren Zeitpunkten (z. B. Prä-Post-Test in Interventionsstudien) treffen, selbst wenn diese kein Ausmaß erreicht, in dem der Schwellenwert zwischen Gesundheit und Krankheit überschritten wird.

\section{Longitudinalstudien}

Die aktuelle Konzeptualisierung von Resilienz als dynamischer Prozess macht den Einsatz längsschnittlicher Untersuchungsdesigns erforderlich. Nur solche Designs erlauben es, Anpassungsprozesse, die in der Veränderung neuraler und/oder kognitiver Funktionen (also Resilienzmechanismen) bestehen, nachzuverfolgen. Idealerweise sind solche Designs auch prospektiv, d.h. psychische Dysfunktionen werden bereits gemessen, bevor die Stressorexposition stattfindet, wodurch sich vorbestehende Dysfunktionen von stressorinduzierten abgrenzen lassen. Der zunehmende Konsens unter Resilienzforschern über einen prospektiv-longitudinalen Ansatz spiegelt sich auch in der Zahl entsprechender Studien wider, die derzeit international verstärkt durchgeführt werden. Beispiele sind die Marine Resiliency Study (USA; [21]), das Police-in-Action-Projekt (Niederlande; [8]), die LORA-Studie (Longitudinal Resilience Assessment) des DFG (Deutsche Forschungsgesellschaft)-Sonderforschungsbereichs 1193 [6] oder das Mainzer Resilienz-Projekt (MARP; [17]; beides in Deutschland).

Longitudinale Studiendesigns sind auch erforderlich, um eindeutigere Aus-

Nervenarzt 2018 $\cdot 89: 747-753$ https://doi.org/10.1007/s00115-018-0529-x

(c) Der/die Autor(en) 2018

A. M. Kunzler · D. A. Gilan · R. Kalisch · O. Tüscher · K. Lieb

Aktuelle Konzepte der Resilienzforschung

Zusammenfassung

Hintergrund. Stressassoziierte psychische Erkrankungen gehören zu den häufigsten und kostenintensivsten Erkrankungen unserer Zeit. Andererseits ist die Erhaltung psychischer Gesundheit angesichts von Stressoren, d. h. Resilienz, ein häufiges Phänomen. Die Erforschung psychischer Resilienz und ihrer zugrunde liegenden Mechanismen bietet innovative Möglichkeiten zur Gesundheitsförderung. Voraussetzung hierfür sind ein einheitliches Verständnis von Resilienz sowie geeignete Methoden der Operationalisierung.

Fragestellung. Moderne Konzepte der Definition, Operationalisierung und Erfassung von Resilienz und deren Implikationen für Studiendesigns in der Resilienzforschung. Material und Methoden. Analyse und Diskussion aktueller Arbeiten und Expertenempfehlungen zur Gestaltung der Resilienzforschung.

Ergebnisse. Die Resilienzforschung befindet sich seit einigen Jahren im
Wandel. Ausgehend von einem neuen Verständnis von Resilienz als dynamischem und veränderbarem Prozess wurden neue Ansätze zur Operationalisierung und Messung vorgeschlagen. Hierzu gehören z. B. ein transdiagnostischer Ansatz und die Identifizierung von Resilienzmechanismen, die Berücksichtigung der Stressorexposition bei der Erfassung des Konstrukts oder längsschnittliche Kohortenstudien.

Diskussion. Durch aktuelle prospektive Longitudinalstudien ist in den kommenden Jahrzehnten mit weiteren gewinnbringenden Erkenntnissen zu rechnen. Eine Herausforderung der zukünftigen Resilienzforschung besteht in der kontinuierlichen Verbreitung der vorgestellten Ansätze.

\section{Schlüsselwörter}

Stressorexposition · Gesundheitsförderung · Resilienzmechanismen · Resilienz-Score · Längsschnittstudien

\section{Current concepts of resilience research}

\section{Abstract}

Background. Stress-related mental disorders are the most prevalent and cost-intensive disorders of our time. On the other hand, the maintenance of mental health despite stressors, i.e. resilience, is a frequent phenomenon. Research on psychological resilience and its underlying mechanisms offers innovative possibilities for health promotion. It requires a consistent understanding of resilience and adequate methods of operationalization. Objectives. Modern concepts of the definition, operationalization and assessment of resilience as well as its implications for study designs in resilience research.

Material and methods. Analysis and discussion of current works and expert recommendations for the design of resilience research.

Results. Resilience research is undergoing a period of transition. Based on a new understanding of resilience as a dynamic and modifiable process, new approaches for operationalization and assessment were proposed. These include, for example, a transdiagnostic approach and the identification of resilience mechanisms, the consideration of stressor exposure in measuring the construct, and longitudinal cohort studies.

Conclusions. In the upcoming decades, further profitable findings from current prospective longitudinal studies can be expected. One challenge for future resilience research consists in the continuous dissemination and implementation of the approaches described.

\section{Keywords}

Stressor exposure $\cdot$ Healthcare promotion . Resilience mechanisms · Resilience score . Longitudinal studies sagen über die (langfristige) Prädiktionskraft einzelner Resilienzfaktoren zu treffen [15]. Zudem wird die psychometrische Güte sog. Resilienz-Skalen i.d. R. querschnittlich überprüft [18, 23], wodurch unklar ist, wie gut diese In- strumente die Resilienz eines Menschen angesichts widriger Lebensumstände prädizieren. Für die Zukunft erscheint daher auch die Validierung von Instrumenten in Längsschnittstudien sinnvoll. 

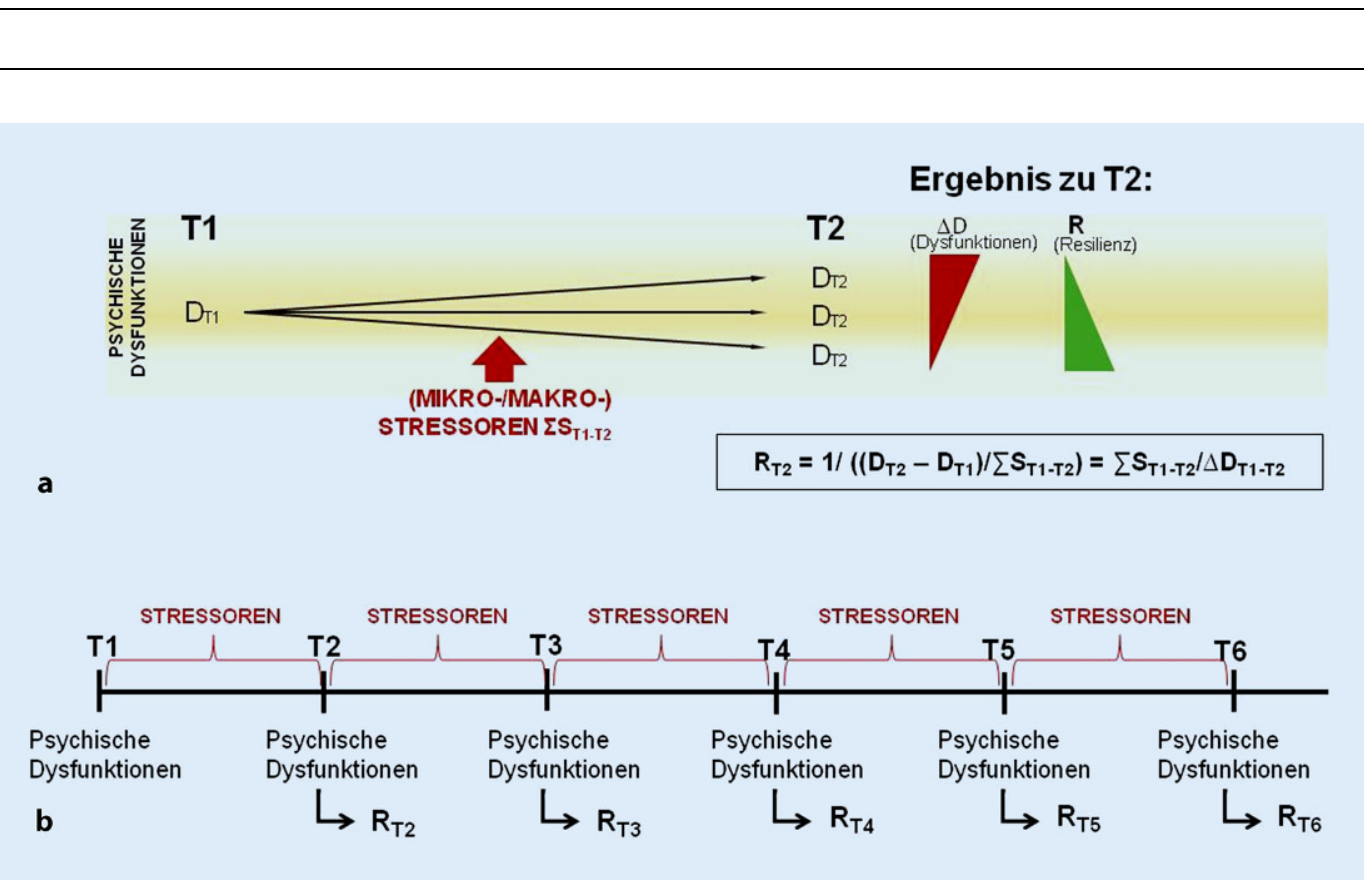

Abb. $2<$ a R-Score zur Messung von Resilienz als Ergebnis. $D$ psychische Dysfunktionen, SStressor, $D_{T 2}-D_{T 1}$ Veränderung in psychischen Dysfunktionen zwischen $\mathrm{T}_{1}$ und $\mathrm{T}_{2,}, \mathrm{~S}_{\mathrm{T} 1-\mathrm{T} 2}$ Stressorload von T1-T2, R Resilienz zu T2. (Adaptiert nach Kalisch et al. [14], mit freundl. Genehmigung Cambridge University Press.) b Längsschnittliches Studiendesign zur Bestimmung des R-Scores zwischen je zwei Messzeitpunkten

\section{Messung von Resilienz}

Auch wenn keine allgemeingültige Resilienzdefinition vorliegt, existiert ein wissenschaftlicher Konsens über zwei grundlegende Elemente des Konzepts:

1. Resilienz erfordert das Vorliegen eines bedeutsamen Stressors und

2. Resilienz besteht in der erfolgreichen Bewältigung dieses Stressors $[7,16]$.

Um die Resilienz eines Menschen valide zu erfassen, erscheint daher die Berücksichtigung der individuellen Stressorexposition dringend erforderlich [14]. Zur Messung von Resilienz als Ergebnis der Anpassung an einen Stressor sollten sowohl die Veränderung der psychischen Gesundheit als auch der individuelle Stressorload (d.h. Art und Ausmaß der Stressoren) in einer gewissen Zeit erfasst werden. Evidenz für die Bedeutung des Stressorloads liefern Studien, die konsistent negative Effekte der Stressorexposition auf die psychische Gesundheit nachwiesen [4].

\section{》) Bislang fehlt ein Goldstan- dard zur Resilienzerfassung}

Ein Goldstandard zur Erfassung von Resilienz wurde bislang nicht etabliert. Derzeit dominiert der Einsatz verschiedener „Resilienz-Skalen“, die auf unterschiedlichen Konzeptualisierungen basieren und häufig querschnittlich eingesetzt werden. Neben Skalen zur Messung von Resilienz als Persönlichkeitseigenschaft erfassen die meisten Instrumente die Verfügbarkeit verschiedener Ressourcen, die zur Aufrechterhaltung bzw. Rückgewinnung der psychischen Gesundheit angesichts widriger Lebensumstände beitragen (vgl. z. B. [23]). Lediglich die Brief Resilience Scale (BRS; $[5,20,23])$ erfasst die Fähigkeit zur Stresserholung und grenzt sich somit von den übrigen Maßen ab. Instrumente, die eine ergebnisorientierte Resilienzerfassung erlauben oder Resilienz als dynamischen Prozess abbilden, liegen bislang nicht vor. Der ResilienzScore, der im Folgenden dargestellt wird, stellt eine Möglichkeit hierfür dar und berücksichtigt die beiden zentralen Elemente des Resilienzkonzepts.

\section{Resilienz-Score zur ergebnis- orientierten Resilienzmessung}

Der Resilienz-Score (R-Score) wurde vorgeschlagen, um Resilienz transdiagnostisch und über quantitative Zielgrößen $\mathrm{zu}$ operationalisieren $[4,13$, 14]. Ausgangspunkt ist ein prospektivlongitudinales Studiendesign mit mindestens zwei Messzeitpunkten $\left(T_{1}, T_{2}\right)$ vor und nach Stressorexposition. Psychische Dysfunktionen zu $\mathrm{T}_{1}$ und $\mathrm{T}_{2}$ werden jeweils über einen Score $\left(D_{T 1}\right.$ und $\mathrm{D}_{\mathrm{T} 2}$ ) ermittelt (s.oben). Die Veränderung in psychischen Dysfunktionen zwischen $T_{1}$ und $T_{2}$ wird als Differenz $\left(D_{T 2}-D_{T 1}\right)$ ausgedrückt, wobei ein positives Vorzeichen eine Zunahme der Dysfunktionen von $\mathrm{T}_{1}$ zu $\mathrm{T}_{2}$ widerspiegelt. Die akkumulierte Stressorexposition einer Person zwischen $T_{1}$ und $T_{2}$ wird ebenfalls über einen geeigneten Score $\left(\sum \mathrm{S}_{\mathrm{T} 1 \text { bis T2 }}\right)$ abgebildet. Um Resilienz als Ergebnis zu operationalisieren, wird anschließend die Veränderung in psychischen Dysfunktionen zwischen $\mathrm{T}_{1}$ und $T_{2}$ am individuellen Stressorload in derselben Zeitspanne normalisiert (Resilienzquotient).

\section{》) Der R-Score ist eine innovative Möglichkeit zur Resilienzerfassung}

Diese ergebnisorientierte Erfassung von Resilienz basiert auf der Annahme, dass eine Person zu $\mathrm{T}_{2}$ umso resilienter ist, je weniger psychische Dysfunktionen sie zwischen $T_{1}$ und $T_{2}$ entwickelt (je kleiner $\left[D_{T_{2}}-D_{T 1}\right]$ ), und dies im Verhältnis zum Stressorload zwischen $T_{1}$ und $T_{2}$ $\left(\sum \mathrm{S}_{\mathrm{T} 1 \text { bis T2 }}\right)$. Demnach wäre eine Person mit hohem Stressorload (hohes $\sum S$ ) und wenigen psychischen Problemen (D) in einer gewissen Zeitspanne resilienter als eine Person, die im gleichen Ausmaß psychische Dysfunktionen zeigt (gleichesD), jedoch nur wenigen Stressoren ausgesetzt ist (niedriges $\sum S$ ). - Abb. 2 veranschaulicht den R-Score in einem longitudinalen 
Studiendesign mit zwei Messzeitpunkten (- Abb. 2a). Dieses Design kann beliebig erweitert werden, wobei sich der R-Score jeweils zwischen zwei Messzeitpunkten bestimmen lässt ( $\bullet$ Abb. 2b). Somit kann Resilienz auch als Prozess über die Zeit ermittelt werden.

Aus diesem Vorschlag ergibt sich die Notwendigkeit, in Längsschnittstudien die Stressorexposition möglichst exakt und quantitativ zu erfassen. Hierbei ist denkbar, qualitativ unterschiedliche Kategorien von Stressoren gesondert $\mathrm{zu}$ erfassen, um auf diese Weise z.B. Resilienz gegenüber den Effekten von Traumatisierungen von Resilienz gegen chronische Belastungen abzugrenzen.

\section{Moderne Methoden der Resilienzforschung}

Durch neue Technologien (z. B. Bildgebung) gewinnt auch die (neuro-)biologische Resilienzforschung zunehmend an Bedeutung (z. B. [9]). Auch wächst das Interesse an einem translationalen Ansatz [19, 25]. Ausgehend von der Grundlagenforschung zur Untersuchung der (z. B. neurophysiologischen) Mechanismen von Resilienz in Tiermodellen werden die Ergebnisse auf die Humanforschung übertragen, um u. a. wirksame Interventionen zur Resilienzförderung abzuleiten.

\section{Fazit für die Praxis}

- Resilienz bezeichnet die Aufrechterhaltung bzw. Rückgewinnung der psychischen Gesundheit angesichts von Stressoren.

- Sie ist keine unveränderliche Persönlichkeitseigenschaft, sondern das Ergebnis eines Anpassungsprozesses.

- Über die Zeit hinweg ist Resilienz dynamisch und veränderbar.

- Neben Resilienzfaktoren rücken zunehmend übergeordnete neurale und kognitive Mechanismen in den Forschungsfokus.

- Aufgrund des Prozesscharakters von Resilienz sind prospektive Längsschnittstudien für die Erforschung des Konzepts erforderlich.

- Moderne Ansätze der Operationalisierung von Resilienz beinhalten eine transdiagnostische, multisystemische und quantitative Vorgehensweise.

- Um Resilienz zu erfassen, sollte die individuelle Stressorexposition berücksichtigt werden. Der R-Score stellt eine Option hierfür dar.

\section{Korrespondenzadresse}

Dipl.-Psych. A. M. Kunzler
Deutsches Resilienz Zentrum
(DRZ) Mainz
Mainz, Deutschland
Angela.Kunzler@unimedizin-
mainz.de

Föderung. Dieses Projekt wurde aus Mitteln des Forschungs- und Innovationsprogramms Horizont 2020 der Europäischen Union im Rahmen der Finanzhilfevereinbarung mit Nr. 777084 und des DFGSonderforschungsbereichs 1193 "Neurobiologie der Resilienz" (Teilprojekte B01, C01) finanziert.

\section{Einhaltung ethischer Richtlinien}

Interessenkonflikt. A.M. Kunzler, D.A. Gilan, R. Kalisch, O. Tüscher und K. Lieb geben an, dass kein Interessenkonflikt besteht.

Dieser Beitrag beinhaltet keine von den Autoren durchgeführten Studien an Menschen oder Tieren.

Open Access. Dieser Artikel wird unter der Creative Commons Namensnennung 4.0 International Lizenz (http://creativecommons.org/licenses/by/4.0/deed. de) veröffentlicht, welche die Nutzung, Vervielfältigung, Bearbeitung, Verbreitung und Wiedergabe in jeglichem Medium und Format erlaubt, sofern Sie den/die ursprünglichen Autor(en) und die Quelle ordnungsgemäßnennen, einen Linkzur Creative Commons Lizenz beifügen und angeben, ob Änderungen vorgenommen wurden.

\section{Literatur}

1. Bengel J, Lyssenko L (2012) Resilienz und psychologische Schutzfaktoren im Erwachsenenalter. Stand der Forschung zu psychologischen Schutzfaktoren von Gesundheit im Erwachsenenalter. Bundeszentrale für gesundheitliche Aufklärung, Köln

2. Bonanno GA, Diminich ED (2013) Annual research review: positive adjustment to adversity -trajectories of minimal-impact resilience and emergent resilience. J Child Psychol Psychiatry 54:378-401

3. Bonanno GA, Westphal M, Mancini AD (2011) Resilience to loss and potential trauma. Annu Rev Clin Psychol 7:511-535

4. Chmitorz A, Kunzler A, Helmreich I et al (2018) Intervention studies to foster resilience - a systematic review and proposal for a resilience framework in future intervention studies. Clin Psychol Rev 59:78-100
5. Chmitorz A, Wenzel M, Stieglitz RD et al (2018) Population-based validation of a German version of the Brief Resilience Scale.PLoSONE 13:e192761

6. Deutsches Resilienz Zentrum (DRZ) Mainz (2017) Aktuelle Studien des DRZ, LORA - Eine Studie zur Erfassung der psychischen Resilienz. https://www.drz-mainz.de/Aktuelle-Studien/\# Lora-Fm_Eine_Studie_Zur_Erfassung_Der Psychischen_Resilienz. Zugegriffen: 12. Febr. 2018

7. Earvolino-Ramirez M (2007) Resilience: a concept analysis. Nurs Forum 42:73-82

8. Experimental Psychopathology and Affective Neuroscience (EPAN) Lab, Donders Institute and Behavioural Science Institute, Radboud University Nijmegen (2018) Police in Action. http://www. epanlab.nl/Research/Main-Projects/Police-inAction/.Zugegriffen: 12. Febr. 2018

9. Feder A, Charney DS, Collins K (2011) Neurobiology of resilience. In: Southwick S, Litz BT, Charney DS, Friedman MJ (Hrsg) Resilience and mental health: challenges across the lifespan. Cambridge University Press, Cambridge, S1-29

10. Gilan DA, Kunzler A, Lieb K (2018) Gesundheitsförderung und Resilienz. PSYCHup2date. https://doi. org/10.1055/s-0043-121606

11. Helmreich I, Kunzler A, Chmitorz A et al (2017) Psychological interventions for resilience enhancement in adults (Protocol). Cochrane Database Syst Rev. https://doi.org/10.1002/14651858.cd012527

12. Hu T, Zhang D, Wang J-L (2015) A meta-analysis of the trait resilience and mental health. Pers Individ Dif 76:18-27

13. Kalisch R, Müller M, Tüscher O (2014) Transdiagnostisch, multisystemisch, und quantitativ. Persönlichkeitsstörungen 18:203-214

14. Kalisch R, Müller M, Tüscher $O$ (2015) A conceptual framework for the neurobiological study of resilience. Behav Brain Sci 38:e92

15. Kalisch R, Baker DG, Basten U et al (2017) The resilienceframeworkas a strategy to combatstressrelated disorders. Nat Hum Behav 1:784-790

16. Luthar SS, Cicchetti D, Becker B (2000) The construct of resilience: a critical evaluation and guidelines for future work. Child Dev 71:543-562

17. Mainzer Resilienz-Projekt (MARP), Focus Program Translational Neurosciences (FTN) of the Johannes Gutenberg University Mainz (2018) http://www. ftn.nic.uni-mainz.de/Marp. Zugegriffen: 12. Febr. 2018

18. Pangallo A,ZibarrasL, Lewis Retal(2015) Resilience through the lens of interactionism: a systematic review. Psychol Assess 27:1-20

19. Scharf SH, Schmidt MV (2012) Animal models of stress vulnerability and resilience in translational research.Curr Psychiatry Rep 14:159-165

20. Smith BW, Dalen J, Wiggins Ket al (2008) The brief resilience scale: assessing the ability to bounce back. Int J Behav Med 15:194-200

21. USDepartment of Veterans Affairs, Health Services Research and Development (2018) Marine Resiliency Study. https://www.hsrd.research.va.gov/ Research/Abstracts.Cfm?Project_Id=2141700196 Zugegriffen: 12. Febr. 2018

22. Vos T, Barber RM, Bell B et al (2015) Global, regional, and national incidence, prevalence, and years lived with disability for 301 acute and chronic diseases and injuries in 188 countries, 1990-2013: a systematic analysis for the Global Burden of Disease Study 2013. Lancet 386:743-780

23. Windle G, Bennett KM, Noyes J (2011) A methodological review of resilience measurement scales. Health Qual Life Outcomes 9:8 
24. World Health Organization (2005) The Bangkok charter for health promotion in a globalized world. World Health Organization, Genf

25. Yehuda R, Flory JD, Southwick S et al (2006) Developing an agenda for translational studies of resilience and vulnerability following trauma exposure. Ann N Y Acad Sci 1071:379-396

\section{G. Mundle \\ Achtsamkeit in der Suchttherapie}

Stuttgart: Kohlhammer 2018, 180 S., (ISBN: 978-3-17-030623-3), 32 EUR

Um den neuen Entwicklungen im Suchtbereich gerecht zu werden, stellen die Herausgeber eine dreiteilige Reihe vor, in denen verschiedene Schwerpunktthemen behan-

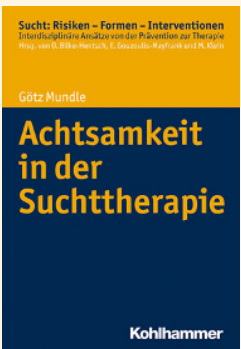
delt werden. In dem von Götz Mundle beschriebenen Band, steht das Thema Achtsamkeit in der Suchttherapie im Fokus. Das Konzept der Achtsamkeit ist in der

Mitte der Gesellschaft angekommen, vor allem jedoch nimmt die Bedeutung von achtsamkeitsbasierenden Therapien im klinischtherapeutischen Bereich zu.

Götz Mundle gelingt eine gute Einführung in die Thematik, in dem er den philosophischen Hintergrund von Achtsamkeit aus östlicher wie westlicher Sicht darstellt. Er beschreibt die Wichtigkeit der eigenen Achtsamkeitspraxis, die eine persönliche Forschungsperspektive auf subjektiver Ebene schulen soll.

Diese nach innen gerichtete Forschung schult das "sich erinnern" (sarati) als "Gewahrsein des Augenblicks", die eingebettet sein sollte in ethische Regeln und einer regelmäßigen, im besten Fall täglichen Praxis. In sanfter, doch klarer Schreibart weist er auf Missverständnisse, Fehlinterpretationen und auch missbräuchliche Auslegungen hin. Die dazu geführt Diskussion, zu Beginn es Buches ist tiefgreifend und bedenkenswert.

Götz Mundle beschreibt die grundlegenden Dimensionen der Achtsamkeit, wie absichtsvoll, gegenwärtig, nicht bewertend und beobachtend. Die Herausforderung für Therapeuten, die mit Achtsamkeitsübungen arbeiten, besteht für inn darin sich auf eine eher ungewöhnliche therapeutische Beziehung einzulassen. Hier können Achtsamkeitsübungen in beiden Richtungen wirksam sein, für den Probanden wie auch für den Therapeuten. Er arbeitet heraus, dass achtsamkeitsorientierte Therapieansätze von einer anderen Therapeutenrolle ausgeht, die durchaus zu Rollenkonflikten führen kann. Götz Mundle differenziert hier gut zwischen achtsamkeitsähnlichen, achtsamkeitsori- entierenden und achtsamkeitsbasierende Psychotherapien. Zentrales Thema ist die Anwendung von Achtsamkeit und Selbstmitgefühl in der Suchttherapie. Im vierten Kapitel folgt eine ausführliche Beschreibung des spezifischen Programms bei Suchterkrankungen, die mindfulness-based-relapse prevention (MBRP). Das Konzept vereint Achtsamkeitsübungen mit kognitiv -verhaltenstherapeutischen Fertigkeiten und Techniken, die auf der traditionellen Rückfallpräventionstherapie beruhen. Es handelt sich hierbei um eine standardisierte 8-wöchige Nachsorgebehandlung, die auf den Strukturen des achtsamkeitsbasierten Stressreduktionsprogramms (MBSR) nach Kabat-Zinn (1990) und der achtsamkeitsbasierten kognitiven Therapie bei Depressionen (MBCT) nach Segal (2012) basiert. Es gibt eine Beschreibung des Stundenablaufs und der durchzuführenden, spezifischen praktischen Übungen. Alle beschriebenen Übungen sind im Anhang, mit vorbereitender Einleitung und durchführender Anleitung zu finden. Eine sehr schöne Sammlung. kurz: richtig gut, lesenswert und erkenntnisfördernd

E. Brügmann (Aachen) 